Berufsverband der Deutschen Urologen e.V. Urologe 2012 $51: 1161$

DOI 10.1007/s00120-012-2978-3

๑) Springer-Verlag 2012
Redaktion

W. Bühmann,Wenningstedt/Sylt

\section{$+\#+$ URO-Telegramm}

Liebe Kolleginnen und Kollegen,

das Uro-Telegramm steht in diesem Heft (fast) ganz unter dem Stern des nahenden DGU-Kongresses in Leipzig - hiermit legen wir Ihnen den Besuch folgender Veranstaltungen vor und während des Kongresses nahe und ans Herz:

\section{Einladung zur ordentlichen} Mitgliederversammlung des BERUFSVERBANDES DER DEUTSCHEN UROLOGEN E.V.

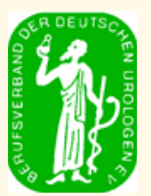

am Freitag, den 28. September 2012 in Leipzig anlässlich des 64. Kongress der Deutschen Gesellschaft für Urologie e.V.

Ort: Congress Centrum Leipzig (CCL) Saal C

Zeit: 12.00 Uhr bis 14.00 Uhr

Tagesordnung:

1. Begrüßung und Feststellung der Beschlussfähigkeit

2. Bericht zur Lage mit Aussprache Präsident: Aktuelle Berufspolitik

1.Vizepräsident: Vertragsworkshop Schriftführer: Neue Versorgungsformen

3. Bericht des Schatzmeisters: Finanzen und Personal

4. Entlastung des Präsidiums

5. Wahlen: 1.Vizepräsident Schriftführer Schatzmeister

6. Bericht der Strukturkommission

7. Bericht aus den Sachausschüssen Referentin des Präsidiums

8. Bericht Bund e.G. Geschäftsführender Vorstand

9. Verschiedenes

Mit freundlichen Grüßen Dr. Axel Schroeder, Präsident

\section{Einladung und Tagesordnung Ordentliche Generalversammlung BUND DER des Bund der Urologen e.G.}

Datum: 27.09. 2012, Uhrzeit :16:15 Uhr

Ort: Leipzig, Congress Center des 64. DGU-Kongress, Raum M1

1. Eröffnung und Begrüßung

2. Bestimmung des Protokollführers

3. Bericht des Vorstandes a) über das Geschäftsjahr 2011

b) über den Jahresabschluss zum 31.12.2011 (Erläuterung)

c) über die zukünftige Entwicklung

d) Verwendung des Jahresergebnisses 2011

4. Bericht des Aufsichtsrates mit Vorstellung und Kenntnisnahme des Prüfungsberichtes des Genossenschaftsverbandes

5. Satzungsänderung zur Einführung eines Marketingentgelts: $§ 14$ Pflichten der Mitglieder

6. Verschiedenes

Dr. A. Münch, Vorsitzender des Aufsichtsrates

\section{Termin-Erinnerung Seminar „Berufsausübungs- BUND DER gemeinschaft"}

Wer von Ihnen, liebe Kolleginnen und Kolleginnen, sich bisher noch nicht zu dem Seminar "Berufsausübungsgemeinschaft" ${ }^{\prime}$, das vom Bund der Urologen veranstaltet wird, angemeldet hat, kann dies für den Ter$\min$

am 8.9.2012 von 10-15.30 Uhr in Potsdam

beim Bund der Urologen, Tel.0621-66003025, E-mail info@bdu-eg.de, nachholen. Dort bekommen Sie auch unter www.bdu-eg.de Informationen zum Inhalt - insbesondere geht es um die Möglichkeiten, die Onkologische Versorgung für urologische Praxen zu erhalten.

Diskutieren Sie mit unserem BDU-Präsidenten Dr. Axel Schroeder im Rahmen der nächsten Online-Sprechstunde

am Donnerstag, 06.09.12, von 20 - 21 Uhr

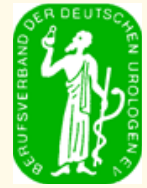

unter www.urologen-im-web.de.

Nutzen Sie die Gelegenheit zum direkten Dialog!

Thema:

Das GKV-Versorgungs-Strukturgesetz: Die wichtigsten Änderungen

- Wirtschaftlichkeitsprüfung

- Heilmittelverordnung

- Regionalisierte Honorarverteilung

- Aktuelles 